\title{
Convergence of a Multigrid Method for Elliptic Equations with Highly Oscillatory Coefficients
}

\author{
Bjorn Engquist * $\quad$ Erding Luo ${ }^{\dagger}$
}

\begin{abstract}
Standard multigrid methods are not so effective for equations with highly oscillatory coefficients. New coarse grid operators based on homogenized operators are introduced to restore the fast convergence rate of multigrid methods. Finite difference approximations are used for the discretization of the equations. The analysis of convergence is based on homogenization theory. Proofs are presented for a two level method with the homogenized coarse grid operator to solve two classes of elliptic equations with Dirichlet boundary conditions.
\end{abstract}

Key Words. elliptic equation, oscillation, finite difference, multigrid method, homogenization theory, convergence

AMS subject classifications. $65 \mathrm{~N} 06,65 \mathrm{~N} 12,65 \mathrm{~N} 55$

* Department of Mathematics, University of California at Los Angeles, LA, CA90024. Research supported by ARPA/ONR N00014-92-J-1890 and NSF DMS91-03104.

${ }^{\dagger}$ IMA, University of Minnesota, Mpls, MN 55455. Research supported by NSF through IMA. 


\section{Introduction}

Consider the multigrid method arising from the finite difference approximations to elliptic equations with highly oscillatory coefficients of the following type

$$
L_{\epsilon} u_{\epsilon}(x)=-\sum_{i, j} \frac{\partial}{\partial x_{i}} a_{i j}^{\epsilon}(x) \frac{\partial}{\partial x_{j}} u_{\epsilon}(x)=f(x),
$$

where $a_{i j}^{\epsilon}(x)=a_{i j}\left(x, \frac{x}{\epsilon}\right), a_{i j}(x, y)=a_{j i}(x, y)$, strictly positive, continuous and 1-periodic in each component of $y$. Also, the operator $L_{\epsilon}$ is uniformly elliptic. That is, there exist two positive constants $q$ and $Q$ independent of $\epsilon$, such that

$$
0<q \sum_{i=1}^{n} \xi_{i}^{2} \leq \sum_{i, j=1}^{n} a_{i j} \xi_{i} \xi_{j} \leq Q \sum_{i=1}^{n} \xi_{i}^{2}
$$

for any $\xi=\left(\xi_{i}\right) \in \Re^{n}$. Here, $\epsilon$ is assumed to be very small, representing the length of the oscillations. These equations have important practical applications, for example, in the study of elasticity and heat conduction for composite materials. One major mathematical technique to deal with these equations is homogenization theory. The theory associates the original equation with its microstructure to some macrostructure effective equation that does not have oscillatory coefficients [2]. By homogenization, as $\epsilon$ gets small, the solution $u_{\epsilon}(x)$ of (1.1) will converge to the solution $u(x)$ of the following homogenized equation,

$$
L_{\mu} u(x)=\sum_{i, j} A_{i j} \frac{\partial^{2} u(x)}{\partial x_{i} \partial x_{j}}=f(x),
$$

where $A_{i j}$ are constants, given by the following expressions,

$$
A_{i j}=\sum_{k} \int_{0}^{1}\left(a_{i j}-a_{i k} \frac{\partial \chi^{j}(y)}{\partial y_{k}}\right) d y .
$$

Here $\chi^{j}(y)$ is 1-periodic in $y$ and satisfies

$$
-\sum_{i, j} \frac{\partial}{\partial y_{i}}\left(a_{i j}(y) \frac{\partial}{\partial y_{j}}\right) \chi^{k}=-\sum_{i} \frac{\partial}{\partial y_{i}} a_{i k}(y) .
$$

Also, the homogenized operator $L_{\mu}$ retains the ellipticity property of the operator $L_{\epsilon}$.

Multigrid methods are usually not so effective when applied to (1.1). Standard construction of coarse grid operators may produce operators with different properties than those 
of the fine grid operators $[1,3,12]$. In order to restore the high efficiency of the multigrid method, a new operator for the coarser grid operator is developed [5, 6]. This operator is called a homogenized coarse grid operator, based on the homogenized form of the equation. For full multigrid or with more general coefficients, the homogenized operator can be numerically calculated from the finer grids based on the local solution of the so called cell problem [5]. For numerical examples on model problems and on the approximation of heat conduction in composite materials [6].

One difficulty for these problems is that the smaller eigenvalues do not correspond to very smooth eigenfunctions. It is thus not easy to represent these eigenfunctions on the coarser grids. After classical smoothing iterations on the fine grid, we know that the high frequency eigenmodes of the errors are reduced, and only the low frequency eigenmodes are significant. Partially following [8], one may realize that the low frequency eigenmodes can be approximated by the corresponding homogenized eigenmodes. This is the reason why effective or homogenized operators are useful when defining the coarse grid operator.

In this paper, using homogenized coarse grid operators, the convergence of the two level method applied to two classes of (1.1) with Dirichlet boundary conditions is analyzed. In chapter 2, we consider the equation with coefficient oscillatory in $x$ direction only; In chapter 3 , we consider the equation with coefficient oscillatory diagonally. We show that as both $\epsilon$ and $h$ go to zeros, our two level multigrid method converges when the number of smoothing iteration $\gamma$ is large enough as a function of $h$. We also require the ratio $h / \epsilon$ not to belong to a small resonance set. More precisely the convergence is proved under the following conditions:

- For the first case in chapter 2 ,

$$
\gamma \geq C h^{-4 / 3} \ln h
$$

- For the second case in chapter 3 ,

$$
\gamma \geq C h^{-5 / 3} \ln h
$$

if $h$ belongs to the set $S\left(\epsilon, h_{0}\right)$ of Diophantine number,

$$
\begin{aligned}
S\left(\epsilon, h_{0}\right)= & \left\{0 \leq h \leq h_{0}|| \frac{k h}{\epsilon}-i|\geq| \frac{\tau}{|k|^{3 / 2}} \mid,\right. \\
& \text { for } \left.\quad i=1,2, \cdots, \quad\left[\frac{k h_{0}}{\epsilon}\right]+1,0 \neq k \in Z\right\},
\end{aligned}
$$


where $S\left(\epsilon, h_{0}\right) \subseteq\left[0, h_{0}\right]\left(h_{0}>0\right)$ with measure $\left|S\left(\epsilon, h_{0}\right)\right| \geq(1-3 \tau) h_{0}$. In [4], Engquist called it the convergence essentially independent of $\epsilon$.

The purpose of this paper is to present new analysis in order to give a theoretical explanation of the computational results presented in $[5,6]$. The bounds on $\gamma$ given above are overly pessimistic compared to the numerical experiments but the $h$ dependence in $\gamma$ exists also in the computations $[5,6]$. The effect of not requiring $h \in S$ is also seen in the numerical tests $[5,9]$.

If the coarse grid operator is defined, i.e., by direct arithmetic averaging an eigenmode analysis of the type given in section 2 and 3 produces the estimate $\gamma=O\left(h^{-2}\right)$. This difference between the correctly homogenized coarse grid operators and other operators is qualitatively consistent with the computational results of $[5,6]$. The $O\left(h^{-2}\right)$ estimate means that there is no multigird effect and the convergence is only produced by the smoothing iterations.

The $l_{2}$ difference between the inverse of the analytic operator $L_{\epsilon}$ and that of the corresponding homogenized operator $L_{\mu}$ is of the order $O(\epsilon)$ [2]. This indicates that an eigenmode analysis of the type used in this paper cannot give estimates better than $\gamma=O\left(h^{-1}\right)$. This is close to the estimate in one space dimension $\gamma \geq C h^{-6 / 5} \ln h$ in [9].

In special cases, it is possible to design prolongation, restriction and coarse grid operators under that the resulting method corresponds to a direct solver [7]. This type of algorithm and methods based on special discretizations with built in a priori knowledge of the oscillatory behavior is outside the scope of this paper.

Since in the sequel of the paper the following lemma is often cited, we introduce it here first.

Lemma 1.1 [4] Suppose $g(x, y) \in C^{3}([0,1] \times[0,1])$ and is 1-periodic in $y$. Let $x_{k}=k h, k=$ $1, \cdots, N$ and $N h=1$. If $h \in S\left(\epsilon, h_{0}\right)$, we have

$$
\left|\sum_{k=1}^{j} g\left(x_{k}, x_{k} / \epsilon\right) h-\int_{0}^{x_{j}} \int_{0}^{1} g(x, y) d y d x\right| \leq C \frac{h}{\tau}, \quad 0 \leq j \leq N .
$$

We always denote the domain $[0,1] \times[0,1]$ by $\bar{\Omega},(0,1) \times(0,1)$ by $\Omega$, and $\bar{\Omega} / \Omega$ by $\partial \Omega$. We discretize the domain by the same number of grid points $N$ with equal step size $h=\frac{1}{N}$ both in $x$ - and $y$-directions. The step size $h$ is chose to belong to the set of $S\left(\epsilon, h_{0}\right)$. And, the ratio of $h$ to the wavelength $\epsilon$ is fixed to be an strictly irrational number. $\bar{\Omega}_{h}$ denotes the set of grid points $(i h, j h) \in \bar{\Omega}, \Omega_{h}$ for $(i h, j h) \in \Omega$, and $\partial \Omega_{h}$ for $(i h, j h) \in \partial \Omega$. $c$ and $C$ present some constants, independent of $\epsilon$ and $h$. $D_{+}^{i}$ and $D_{-}^{i}$ are standard forward and backward finite difference operators in $x$ direction; $D_{+}^{j}$ and $D_{-}^{j}$ are for $y$ direction. $\|\cdot\|_{h}$ represents the discrete $L_{2}$-norm, indexed from 1 to $N-1$. 


\section{Oscillation Along a Coordinate Direction}

\subsection{Model Equation}

Consider a special case of (1.1), a two-dimensional elliptic problem with coefficients oscillatory in $x$-direction only,

$$
\begin{aligned}
-\frac{\partial}{\partial x} a_{\epsilon}(x) \frac{\partial \phi_{\epsilon}}{\partial x}-\frac{\partial}{\partial y} a_{\epsilon}(x) \frac{\partial \phi_{\epsilon}}{\partial y} & =f(x, y), \quad(x, y) \in \Omega, \\
\phi_{\epsilon}(x, y) & =0, \quad(x, y) \in \partial \Omega,
\end{aligned}
$$

where $a_{\epsilon}(x)$ is a strictly positive continuous function, and $a_{\epsilon}(x)=a(x / \epsilon)=a(x / \epsilon+1)$. Also, the operator satisfies the property of (1.2). From (1.3), the corresponding homogenized equation of (2.1) is:

$$
\begin{aligned}
-\mu \frac{\partial^{2} \phi}{\partial x^{2}}-\bar{a} \frac{\partial^{2} \phi}{\partial y^{2}} & =f(x, y), \quad(x, y) \in \Omega, \\
\phi(x, y) & =0, \quad(x, y) \in \partial \Omega,
\end{aligned}
$$

where $\mu=\left(\int_{0}^{1} 1 / a(x) d x\right)^{-1}$ and $\bar{a}=\int_{0}^{1} a(x) d x$ are the harmonic and arithmetical averages respectively.

As $\epsilon$ goes to zero, we know that the solution $\phi_{\epsilon}$ of $(2.1)$ converges to the solution $\phi$ of $(2.2)$.

Now, consider a corresponding discretized equation of (2.1),

$$
-D_{+}^{i} a_{i} D_{-}^{i} u_{i j}^{h}-D_{+}^{j} b_{i} D_{-}^{j} u_{i j}^{h}=f_{i j}^{h}, \quad(i, j) \in \Omega_{h}
$$

where $a_{i}=a_{\epsilon}\left(x_{i}-\frac{h}{2}\right), b_{i}=a_{\epsilon}\left(x_{i}\right), \quad i=1, \cdots, N$.

Denote the discretization of the homogenized operator $-\mu \frac{\partial^{2}}{\partial x^{2}}-\bar{a} \frac{\partial^{2}}{\partial y^{2}}$ in (2.2) by

$$
L_{\mu, h}=-\mu_{h} D_{+}^{i} D_{-}^{i}-b_{h} D_{+}^{j} D_{-}^{j},
$$

where $\mu_{h}=\left(h \sum_{i=1}^{N} \frac{1}{a_{i}}\right)^{-1}, b_{h}=\sum_{i=1}^{N} b_{i} h$. The operator of the two level method by using the homogenized coarse grid operator $[5,6]$ can be expressed as

$$
M=\left(I-I_{H}^{h} L_{H}^{-1} I_{h}^{H} L_{\epsilon, h}\right) S^{\gamma},
$$

For simplicity, in the sequel of the paper, $I_{h}^{H}$ and $I_{H}^{h}$ always denote the weighting restriction and bilinear interpolation respectively, and the smoothing operator $S$,

$$
S=I-\alpha L_{\epsilon, h},
$$

where $\alpha$ is the inverse of the largest eigenvalue of $L_{\epsilon, h}$, has order of $h^{-2} . L_{H}$ is taken to be the corresponding homogenized operator $L_{\mu, H}$. 


\subsection{Convergence Analysis}

Instead of the operator $M$, we consider a simplified operator, denoted by $M_{1}$,

$$
M_{1}=\left(I-L_{\mu, h}^{-1} L_{\epsilon, h}\right)\left(I-\alpha L_{\epsilon, h}\right)^{\gamma}=\left(I-L_{\mu, h}^{-1} L_{\epsilon, h}\right) S^{\gamma} .
$$

Theorem 2.1 If the ratio of $h$ to $\epsilon$ is fixed, $h$ belongs to the set of Diophantine number $S\left(\epsilon, h_{0}\right)$ defined in (1.6), then there exist two constants $C$ and $\rho_{0}$ such that

$$
\left\|M_{1}\right\|_{h} \leq \rho_{0}<1
$$

whenever $\gamma \geq C h^{-1-1 / 3} \ln (h)$.

Let's introduce some lemmate first, which are used in the proof of Theorem 2.1.

Lemma 2.1 Assume

$$
Z_{i}=\frac{h}{\epsilon}\left(i-\mu_{h} \sum_{k=1}^{i} \frac{1}{a_{k}}\right), \quad i=0, \cdots, N .
$$

Then, $Z_{i}$ is bounded, and satisfies

$$
L_{\epsilon, h} Z_{i}=-\frac{1}{\epsilon} D_{+}^{i} a_{i}, \quad i=1, \cdots, N-1 .
$$

Proof. By Lemma 1.1, taking $j=N$, we have

$$
\left|\mu_{h}^{-1}-\int_{0}^{1} \frac{1}{a(y)} d y\right| \leq C h .
$$

Hence,

$$
\frac{h}{2 \epsilon}\left(i-\mu_{h} \sum_{k=1}^{i} \frac{1}{a_{k}}\right)=\frac{1}{2 \epsilon}\left(\frac{i h}{\mu_{h}}-i h \int_{0}^{1} \frac{1}{a(y)} d y-\sum_{k=1}^{i} \frac{1}{a_{k}} h+i h \int_{0}^{1} \frac{1}{a(y)} d y\right)=O(1) .
$$

$Z_{i}$ is bounded for $i=0, \cdots, N$. Also, $Z_{i}$ satisfies the following equation,

$$
a_{i}\left(1-\epsilon D_{-}^{i} Z_{i}\right)=\mu_{h}, \quad i=1, \cdots, N-1 .
$$

Lemma 2.2 Assume $\eta_{i}$ satisfies

$$
\begin{aligned}
-\epsilon D_{-}^{i} \eta_{i} & =b_{i}-b_{h}, \quad i=1, \cdots, N, \\
\eta_{0} & =0 .
\end{aligned}
$$

Then, $\eta_{i}$ is bounded. 
Proof. Directly applying Lemma 1.1, we can establish the result.

Lemma 2.3 Assume $U_{i j}$ satisfies

$$
\begin{aligned}
L_{\mu, h} U_{i j} & =\lambda_{\epsilon} \phi_{i j}^{h}, \quad(i, j) \in \Omega_{h}, \\
U_{i j} & =0, \quad(i, j) \in \partial \Omega_{h},
\end{aligned}
$$

where $\left(\lambda_{\epsilon}, \phi_{i j}^{h}\right)$ is a normalized eigenpair of $L_{\epsilon, h}$. That is,

$$
\begin{aligned}
L_{\epsilon, h} \phi_{i j}^{h} & =\lambda_{\epsilon} \phi_{i j}^{h}, \quad(i, j) \in \Omega_{h}, \\
\phi_{i j}^{h} & =0, \quad(i, j) \in \partial \Omega_{h} .
\end{aligned}
$$

Then,

$$
\begin{gathered}
\|U\|_{1}^{2}=\sum_{i, j=1}^{N}\left(D_{-}^{i} U_{i j} h\right)^{2}=O\left(\lambda_{\epsilon}\right) \\
\sum_{i, j=1}^{N-1}\left(\left(D_{+}^{i} D_{-}^{i} U_{i j}\right)^{2}+\left(D_{-}^{i} D_{-}^{j} U_{i j}\right)^{2}+\left(D_{+}^{j} D_{-}^{j} U_{i j}\right)^{2}\right) h^{2}=O\left(\lambda_{\epsilon}^{2}\right) .
\end{gathered}
$$

Proof. Multiplied by $U_{i j}$,

$$
L_{\mu, h} U_{i j}=L_{\epsilon, h} \phi_{i j}^{h},
$$

added by parts, (2.9) then follows.

For (2.10), note first for any grid function $U_{i j}$, vanishing on $\partial \Omega_{h}$, we have

$$
\begin{aligned}
& \sum_{i, j=1}^{N-1}\left(D_{+}^{i} D_{-}^{i} U_{i j}\right)\left(D_{+}^{j} D_{-}^{j} U_{i j}\right)=\sum_{i, j=1}^{N}\left(D_{+}^{i} D_{-}^{i} U_{i j}\right)\left(D_{+}^{j} D_{-}^{j} U_{i j}\right) \\
= & \sum_{i, j=1}^{N}\left(D_{-}^{i} D_{-}^{j} U_{i j}\right)^{2} \geq 0 .
\end{aligned}
$$

Multiply $D_{+}^{i} D_{-}^{i} U_{i j}$ on both sides of (2.8), and take summation,

$$
\begin{aligned}
& \sum_{i, j=1}^{N-1} \mu_{h}\left(D_{+}^{i} D_{-}^{i} U_{i j}\right)^{2} \\
\leq & \sum_{i, j=1}^{N-1} \mu_{h}\left(D_{+}^{i} D_{-}^{i} U_{i j}\right)^{2}+\sum_{i, j=1}^{N-1} b_{h}\left(D_{+}^{i} D_{-}^{i} U_{i j}\right)\left(D_{+}^{j} D_{-}^{j} U_{i j}\right) \\
= & \sum_{i, j=1}^{N-1} \lambda_{\epsilon} \phi_{i j}\left(D_{+}^{i} D_{-}^{i} U_{i j}\right) .
\end{aligned}
$$


Then, we can establish

$$
\sum_{i, j=1}^{N-1} \mu_{h}\left(D_{+}^{i} D_{-}^{i} U_{i j}\right)^{2} \leq C \lambda_{\epsilon}^{2},
$$

for some constant $C$. Analogously, we can get

$$
\sum_{i, j=1}^{N-1} \mu_{h}\left(D_{+}^{j} D_{-}^{j} U_{i j}\right)^{2} \leq C \lambda_{\epsilon}^{2} .
$$

(2.11), (2.12) and (2.13) complete the proof.

Lemma 2.4 Assume $\phi_{i j}^{h}, U_{i j}$ are defined in Lemma D.马. Then,

$$
\left\|\phi^{h}-U\right\|_{h}=O\left(\epsilon \lambda_{\epsilon}\right)
$$

Proof. Introduce the following discrete function

$$
G_{i j}=U_{i j}-\epsilon Z_{i} D_{-}^{i} U_{i j}-\phi_{i j}^{h}, \quad(i, j) \in \bar{\Omega}_{h},
$$

where $Z_{i}$ is defined in Lemma 2.1. Such $G_{i j}$ vanishes at boundary, i.e.,

$$
G_{i j}=0, \quad(i, j) \in \partial \Omega_{h}
$$

By calculation, we have

$$
\begin{aligned}
& L_{\epsilon, h} G_{i j} \\
= & -D_{-}^{i}\left(\epsilon a_{i+1} Z_{i} D_{+}^{i} D_{-}^{i} U_{i j}\right)-\epsilon b_{i} Z_{i} D_{+}^{j} D_{-}^{j} D_{-}^{i} U_{i j}+\left(b_{h}-b_{i}\right) D_{+}^{j} D_{-}^{j} U_{i j} \\
= & -D_{-}^{i}\left(\epsilon a_{i+1} Z_{i} D_{+}^{i} D_{-}^{i} U_{i j}\right)-\epsilon b_{i} Z_{i} D_{+}^{j} D_{-}^{j} D_{-}^{i} U_{i j}+\epsilon D_{-}^{i} \eta_{i} D_{+}^{j} D_{-}^{j} U_{i j},
\end{aligned}
$$

where $(i, j) \in \Omega_{h}$, and $\eta_{i}$ is defined in Lemma 2.2. Multiply $G_{i j}$ on both sides, and take summation,

$$
\begin{aligned}
& \sum_{i, j=1}^{N} L_{\epsilon, h} G_{i j} G_{i j} h^{2} \\
= & \epsilon\left(\sum_{i, j=1}^{N-1} a_{i+1} Z_{i} D_{+}^{i} D_{-}^{i} U_{i j} D_{+}^{i} G_{i j}+\sum_{i=1}^{N-1} \sum_{j=0}^{N-1} b_{i} Z_{i} D_{+}^{j} D_{-}^{i} U_{i j} D_{+}^{j} G_{i j}\right. \\
& \left.-\sum_{i, j=1}^{N-1} \eta_{i} D_{+}^{j} D_{-}^{j} U_{i j} D_{+}^{i} G_{i j}-\sum_{i, j=1}^{N} \eta_{i-1} D_{-}^{i} D_{-}^{j} D_{+}^{j} U_{i j} G_{i j}\right) h^{2}
\end{aligned}
$$




$$
\begin{aligned}
= & \epsilon\left(\sum_{i, j=1}^{N-1} a_{i+1} Z_{i} D_{+}^{i} D_{-}^{i} U_{i j} D_{+}^{i} G_{i j}+\sum_{i=1}^{N-1} \sum_{j=0}^{N-1} b_{i} Z_{i} D_{+}^{j} D_{-}^{i} U_{i j} D_{+}^{j} G_{i j}\right. \\
& \left.-\sum_{i, j=1}^{N-1} \eta_{i} D_{+}^{j} D_{-}^{j} U_{i j} D_{+}^{i} G_{i j}+\sum_{i=1}^{N-1} \sum_{j=0}^{N-1} \eta_{i} D_{+}^{i} D_{+}^{j} U_{i j} D_{+}^{j} G_{i+1 j}\right) h^{2} \\
\leq & C \epsilon\left(\sqrt{\left.\sum_{i, j=1}^{N-1}\left(D_{+}^{i} D_{-}^{i} U_{i j} h\right)^{2}+\sum_{i, j=1}^{N-1}\left(D_{+}^{j} D_{-}^{j} U_{i j} h\right)^{2}+\sum_{i, j=1}^{N}\left(D_{-}^{j} D_{-}^{i} U_{i j} h\right)^{2}\right)}\right. \\
& \times \sqrt{\sum_{i, j=1}^{N}\left(D_{-}^{i} G_{i j} h\right)^{2}+\left(D_{-}^{j} G_{i j} h\right)^{2}} .
\end{aligned}
$$

By Lemma 2.3, we have

$$
\sum_{i, j=1}^{N} L_{\epsilon, h} G_{i j} G_{i j} h^{2} \leq C \epsilon \lambda_{\epsilon} \sqrt{\sum_{i, j=1}^{N}\left(D_{-}^{i} G_{i j} h\right)^{2}+\left(D_{-}^{j} G_{i j} h\right)^{2}} .
$$

Hence,

$$
\sqrt{\sum_{i, j=1}^{N}\left(D_{-}^{i} G_{i j} h\right)^{2}+\left(D_{-}^{j} G_{i j} h\right)^{2}} \leq O\left(\epsilon \lambda_{\epsilon}\right)
$$

By Poincare inequality,

$$
\|G\|_{h}=O\left(\epsilon \lambda_{\epsilon}\right) .
$$

By Lemma 2.1 and Lemma 2.3,

$$
\left\|\epsilon Z D_{-}^{i} U\right\|_{h} \leq \epsilon \max _{0 \leq i \leq N}\left|Z_{i}\right|\|U\|_{1} \leq C \epsilon \sqrt{\lambda_{\epsilon}} .
$$

Hence,

$$
\left\|\phi^{h}-U\right\|_{h} \leq\|G\|_{h}+\left\|\epsilon Z D_{-}^{i} U\right\|_{h} \leq C \epsilon \lambda_{\epsilon} .
$$

Meanwhile, the inequality tells us that

$$
\left\|\left(I-L_{\mu, h}^{-1} L_{\epsilon, h}\right) \phi^{h}\right\|_{h} \leq C \epsilon \lambda_{\epsilon} .
$$

The proof of lemma is completed.

We are now able to show Theorem 2.1. 
Proof of Theorem 2.1. Denote the eigenvalues of $L_{\epsilon, h}$ and $\Delta_{h}$ (Laplacian operator) by $\lambda_{i j}^{\epsilon}$, $\lambda_{i j}$, respectively, where $i, j=1, \cdots, N-1$. Then, by dividing the set of eigenvalues into two subsets, say $\left\{\lambda_{i j}^{\epsilon}, \quad i^{2}+j^{2} \leq k_{0}^{2}\right\}$ and $\left\{\lambda_{i j}^{\epsilon}, \quad(N-1)^{2} \geq i^{2}+j^{2} \geq k_{0}^{2}\right\}$ for some $k_{0}$, we can split the complete eigenspace of $L_{\epsilon, h}$ into two orthogonal subspaces. Namely, the space of low frequency expanded by the eigenfunctions whose corresponding eigenvalues belong to the first set, and that of high frequency expanded by the eigenfunctions whose corresponding eigenvalues belong to the second set. By minimax principle of eigenvalues, it is easy to see that

$$
c \lambda_{i j} \leq \lambda_{i j}^{\epsilon} \leq C \lambda_{i j}
$$

for some constants $c$ and $C$.

For any normalized vector $\xi$ such that

$$
\xi=\Sigma_{i, j=1}^{N-1} \beta_{i j} \phi_{i j}^{\epsilon}
$$

where $\Sigma_{i, j=1}^{N-1} \beta_{i j}^{2}=1$, we have

$$
\begin{aligned}
M_{1} \xi & =\Sigma_{i, j=1}^{N-1} \beta_{i j} M_{1} \phi_{i j}^{\epsilon} \\
& =\Sigma_{i^{2}+j^{2} \leq k_{0}^{2}} \beta_{i j} M_{1} \phi_{i j}^{\epsilon}+\Sigma_{i^{2}+j^{2}>k_{0}^{2}} \beta_{i j} M_{1} \phi_{i j}^{\epsilon} .
\end{aligned}
$$

Thus,

$$
\left\|M_{1} \xi\right\|_{h} \leq\left\|\Sigma_{i^{2}+j^{2} \leq k_{0}^{2}} \beta_{i j} M_{1} \phi_{i j}^{\epsilon}\right\|_{h}+\left\|\Sigma_{i^{2}+j^{2}>k_{0}^{2}} \beta_{i j} M_{1} \phi_{i j}^{\epsilon}\right\|_{h} .
$$

In the following analysis, we consider (2.17) in two steps.

Step 1: Low frequency subspace.

$$
\begin{aligned}
I_{1} & =\left\|\Sigma_{i^{2}+j^{2} \leq k_{0}^{2}} \beta_{i j} M_{1} \phi_{i j}^{\epsilon}\right\|_{h} \\
& =\left\|\Sigma_{i^{2}+j^{2} \leq k_{0}^{2}} \beta_{i j}\left(I-L_{\mu, h}^{-1} L_{\epsilon, h}\right)\left(I-\alpha L_{\epsilon, h}\right)^{\gamma} \phi_{i j}^{\epsilon}\right\|_{h} \\
& \leq \Sigma_{i^{2}+j^{2} \leq k_{0}^{2}} \mid \beta_{i j}\left\|\left(I-L_{\mu, h}^{-1} L_{\epsilon, h}\right) \phi_{i j}^{\epsilon}\right\|_{h} .
\end{aligned}
$$

By Lemma 2.4,

$$
\left\|\left(I-L_{\mu, h}^{-1} L_{\epsilon, h}\right) \phi_{i j}^{\epsilon}\right\|_{h} \leq C \epsilon \lambda_{i j}^{\epsilon} \leq C \epsilon \lambda_{i j} .
$$

The corresponding eigenvalue of Laplacian operator $\Delta_{h}$ can be explicitly expressed as

$$
\lambda_{i j}=\frac{4}{h^{2}}\left(\sin ^{2}\left(\frac{\pi}{2} i h\right)+\sin ^{2}\left(\frac{\pi}{2} j h\right)\right), \quad i, j=1, \cdots, N-1 .
$$

By Taylor expansion, it follows that

$$
\lambda_{i j}=\left(i^{2}+j^{2}\right)\left(C+O\left((i h)^{2}+(j h)^{2}\right)\right)
$$


Hence,

$$
I_{1} \leq C \epsilon \Sigma_{i^{2}+j^{2} \leq k_{0}^{2}}\left|\beta_{i j}\right|\left(i^{2}+j^{2}\right) .
$$

By the constraint $\Sigma_{i^{2}+j^{2} \leq k_{0}^{2}} \beta_{i j}^{2} \leq 1$,

$$
I_{1} \leq C \epsilon k_{0}^{3} \text {. }
$$

Since the ratio of $h$ to $\epsilon$ is fixed to be an irrational number, $h$ has the same order as $\epsilon$. We have

$$
I_{1} \leq C h k_{0}^{3} \text {. }
$$

Therefore, in order to make $I_{1}<\frac{1}{2}$, it's sufficient to have

$$
k_{0} \leq C h^{-1 / 3}
$$

Step 2: High frequency subspace.

$$
\begin{aligned}
I_{2} & =\left\|\Sigma_{i^{2}+j^{2}>k_{0}^{2}} \beta_{i j} M_{1} \phi_{i j}^{\epsilon}\right\|_{h} \\
& \leq\left\|L_{\epsilon, h}^{-\frac{1}{2}}\left(I-L_{\epsilon, h}^{\frac{1}{2}} L_{\mu, h}^{-1} L_{\epsilon, h}^{\frac{1}{2}}\right)\right\|_{h}\left\|L_{\epsilon, h}^{\frac{1}{2}}\left(1-\alpha L_{\epsilon, h}\right)^{\gamma}\left(\Sigma_{i^{2}+j^{2}>k_{0}^{2}} \beta_{i j} \phi_{i j}^{\epsilon}\right)\right\|_{h} .
\end{aligned}
$$

Since $\left\|L_{\epsilon, h}^{-\frac{1}{2}}\right\|_{h} \leq C$ and $\left\|I-L_{\epsilon, h}^{\frac{1}{2}} L_{\mu, h}^{-1} L_{\epsilon, h}^{\frac{1}{2}}\right\|_{h} \leq C$,

$$
\begin{aligned}
I_{2} & \leq C \sqrt{\sum_{i^{2}+j^{2}>k_{0}^{2}} \beta_{i j}^{2}\left\|L_{\epsilon, h}^{\frac{1}{2}}\left(1-\alpha L_{\epsilon, h}\right)^{\gamma} \phi_{i j}^{\epsilon}\right\|_{h}^{2}} \\
& \leq C \max _{k_{0}^{2} \leq \lambda \leq \lambda_{N-1 N-1}^{\epsilon}}\left|\lambda^{\frac{1}{2}}(1-\alpha \lambda)^{\gamma}\right| .
\end{aligned}
$$

Assume $1+2 \gamma>\frac{1}{\alpha k_{0}^{2}}=\frac{\lambda_{N-1 N-1}^{\epsilon}}{k_{0}^{2}}\left(\geq C \frac{h^{-2}}{k_{0}^{2}}\right)$, we have

$$
I_{2} \leq C k_{0}\left(1-\alpha k_{0}^{2}\right)^{\gamma} \text {. }
$$

For $I_{2} \leq \frac{1}{2}$, it is sufficient to have

$$
\gamma \geq C \frac{h^{-2}}{k_{0}^{2}} \ln k_{0}\left(\geq \frac{1}{\alpha k_{0}^{2}}\right) .
$$

Combining (2.21) and (2.24), we have

$$
\gamma \geq C h^{-1-\frac{1}{3}} \ln h .
$$

The proof is completed.

Now, we are ready to show the main result. 
Theorem 2.2 There exists constant $C$, the operator $M$ defined by (2.5) satisfies

$$
\rho(M) \leq \rho_{0}<1
$$

whenever $h$ belongs to the set $S\left(\epsilon, h_{0}\right)$ of Diophantine number, and

$$
\gamma \geq C h^{-1-1 / 3} \ln h
$$

Before we carry out the proof, we need the following lemma. Let

$$
M_{2}=\left(L_{\mu, h}^{-1}-I_{H}^{h} L_{H}^{-1} I_{h}^{H}\right) L_{\epsilon, h} S^{\gamma} .
$$

Lemma 2.5 For some constant $C$,

$$
\left\|M_{2}\right\|_{h} \leq \frac{C}{\gamma}
$$

Proof. Since $L_{\mu, h}$ and $L_{H}=L_{\mu, H}$ are the homogenized operators defined respectively on fine and coarser grid with constant coefficients, they are well behaved [11, 12]. Furthermore,

$$
\left\|L_{\mu, h}^{-1}-I_{H}^{h} L_{H}^{-1} I_{h}^{H}\right\|_{h} \leq C h^{2} .
$$

Therefore,

$$
\begin{aligned}
\left\|M_{2}\right\|_{h} & \leq\left\|L_{\mu, h}^{-1}-I_{H}^{h} L_{H}^{-1} I_{h}^{H}\right\|_{h}\left\|L_{\epsilon, h} S^{\gamma}\right\|_{h} \\
& \leq C h^{2} h^{-2} / \gamma \\
& \leq \frac{C}{\gamma} .
\end{aligned}
$$

Proof of Theorem 2.2. Note that

$$
M=M_{1}+M_{2}
$$

Therefore,

$$
\|M\|_{h} \leq\left\|M_{1}\right\|_{h}+\left\|M_{2}\right\|_{h}
$$

Since $\rho(M) \leq\|M\|_{h}$, by Theorem 2.1 and Lemma 2.5, the rest of the proof can easily follow. 


\section{Oscillation Along the Diagonal Direction}

\subsection{Model Equation}

Consider another special case of (1.1), a two-dimensional elliptic problem with coefficients oscillatory diagonally,

$$
\begin{aligned}
-\frac{\partial}{\partial x} a_{\epsilon}(x-y) \frac{\partial \phi_{\epsilon}}{\partial x}-\frac{\partial}{\partial y} a_{\epsilon}(x-y) \frac{\partial \phi_{\epsilon}}{\partial y} & =f(x, y), \quad(x, y) \in \Omega \\
\phi_{\epsilon}(x, y) & =0, \quad(x, y) \in \partial \Omega
\end{aligned}
$$

where $a_{\epsilon}(x)$ is a strictly positive continuous function, and $a_{\epsilon}(x)=a(x / \epsilon)=a(x / \epsilon+1)$. Also, the operator has the property of (1.2). From (1.3), the corresponding homogenized equation of (3.1) is:

$$
\begin{aligned}
-\frac{\bar{a}+\mu}{2} \frac{\partial^{2} \phi}{\partial x^{2}}+(\bar{a}-\mu) \frac{\partial^{2} \phi}{\partial x \partial y}-\frac{\bar{a}+\mu}{2} \frac{\partial^{2} \phi}{\partial y^{2}} & =f(x, y), \quad(x, y) \in \Omega \\
\phi(x, y) & =0, \quad(x, y) \in \partial \Omega
\end{aligned}
$$

where $\bar{a}=\int_{0}^{1} a(x) d x$, and $\mu=\left(\int_{0}^{1} 1 / a(x) d x\right)^{-1}$.

As $\epsilon$ goes to zero, we know that the solution $\phi_{\epsilon}$ of (3.1) converges to the solution $\phi$ of (3.2).

Now, consider a corresponding discretized equation of (3.1),

$$
-D_{+}^{i} a_{i j} D_{-}^{i} u_{i j}^{h}-D_{+}^{j} b_{i j} D_{-}^{j} u_{i j}^{h}=f_{i j}^{h}, \quad(i, j) \in \Omega_{h}
$$

where

$$
a_{i j}=a\left(\frac{x_{i}-h / 2-y_{j}}{\epsilon}\right), \quad b_{i j}=a\left(\frac{x_{i}-y_{j}+h / 2}{\epsilon}\right), \quad(i, j) \in \bar{\Omega}_{h} .
$$

Here, we assume the discretized coefficients have the following property,

$$
a_{j 0}=b_{0 N-j+1}, \quad j=0, \cdots, N .
$$

Denote the discretization of the homogenized operator

$$
-\frac{\bar{a}+\mu}{2} \frac{\partial^{2}}{\partial x^{2}}+(\bar{a}-\mu) \frac{\partial^{2}}{\partial x \partial y}-\frac{\bar{a}+\mu}{2} \frac{\partial^{2}}{\partial y^{2}}
$$

in (3.2) by

$$
L_{\mu, h}=-\frac{\mu_{h}+a_{h}}{2}\left(D_{+}^{i} D_{-}^{i}+D_{+}^{j} D_{-}^{j}\right)+\frac{\mu_{h}-a_{h}}{2}\left(D_{+}^{i} D_{+}^{j}+D_{-}^{i} D_{-}^{j}\right),
$$


where $\mu_{h}=\left(h \sum_{k=1}^{N} \frac{1}{a_{k 0}}\right)^{-1}$, and $a_{h}=h \sum_{k=1}^{N} a_{k 0}$.

The operator of the two level method can be expressed as

$$
M=\left(I-I_{H}^{h} L_{H}^{-1} I_{h}^{H} L_{\epsilon, h}\right) S^{\gamma}=\left(I-I_{H}^{h} L_{H}^{-1} I_{h}^{H} L_{\epsilon, h}\right)\left(I-\alpha L_{\epsilon, h}\right)^{\gamma},
$$

where $\alpha$ is the inverse of the largest eigenvalue of $L_{\epsilon, h}$, has order of $h^{-2}$. And $L_{H}=L_{\mu, H}$.

\subsection{Convergence Analysis}

We still first consider the simplified operator $M_{1}$ defined in (2.6).

Theorem 3.1 If the ratio of $h$ to $\epsilon$ is strictly irrational, $h$ belongs to the set of Diophantine number $S\left(\epsilon, h_{0}\right)$ defined in (1.6), then there exist two constants $C$ and $\rho_{0}$ such that

$$
\left\|M_{1}\right\|_{h} \leq \rho_{0}<1
$$

whenever $\gamma \geq C h^{-1-2 / 3} \ln (h)$.

In order to prove Theorem 3.1, we introduce the following lemmate first.

Lemma 3.1 Define two discrete functions on $\bar{\Omega}_{h}$,

$$
\begin{array}{ll}
Z_{i j}^{1}=\frac{h}{2 \epsilon}\left(i-j-\mu_{h} \sum_{k=1}^{i} \frac{1}{a_{k j}}+\mu_{h} \sum_{k=1}^{j} \frac{1}{b_{0 k}}\right), \quad(i, j) \in \bar{\Omega}_{h}, \\
Z_{i j}^{2}=\frac{h}{2 \epsilon}\left(j-i+\mu_{h} \sum_{k=1}^{i} \frac{1}{a_{k 0}}-\mu_{h} \sum_{k=1}^{j} \frac{1}{b_{i k}}\right), \quad(i, j) \in \bar{\Omega}_{h} .
\end{array}
$$

Then, $Z_{i j}^{1}, Z_{i j}^{2}$ are bounded, and satisfy

$$
L_{\epsilon, h} Z_{i j}^{1}=-\frac{1}{\epsilon} D_{+}^{i} a_{i j}, \quad L_{\epsilon, h} Z_{i j}^{2}=-\frac{1}{\epsilon} D_{+}^{j} b_{i j},
$$

for $(i, j) \in \Omega_{h}$.

Proof. Notice that by the assumption of the coefficients,

$$
\sum_{k=1}^{N} \frac{h}{a_{k j}}=\sum_{k=1}^{N} \frac{h}{b_{i k}}=\mu_{h}^{-1}, \quad(i, j) \in \bar{\Omega}_{h} .
$$


Applying the operator $L_{\epsilon, h}$ to $Z_{i j}^{1}$, (3.9) then follows. Now, we write $Z_{i j}^{1}$ as follows,

$$
Z_{i j}^{1}=\frac{h}{2 \epsilon}\left(i-\mu_{h} \sum_{k=1}^{i} \frac{1}{a_{k j}}\right)-\frac{h}{2 \epsilon}\left(j-\mu_{h} \sum_{k=1}^{j} \frac{1}{b_{0 k}}\right) .
$$

By Lemma 1.1,

$$
\frac{h}{2 \epsilon}\left(i-\mu_{h} \sum_{k=1}^{i} \frac{1}{a_{k j}}\right)=O(1), \quad \frac{h}{2 \epsilon}\left(j-\mu_{h} \sum_{k=1}^{j} \frac{1}{b_{0 k}}\right)=O(1) .
$$

Hence, $Z_{i j}^{1}$ is bounded for $(i, j) \in \bar{\Omega}_{h}$, and satisfies

$$
a_{i j}\left(1-\epsilon D_{-}^{i} Z_{i j}^{1}\right)=\frac{a_{i j}+\mu_{h}}{2}, \quad-\epsilon b_{i j} D_{-}^{j} Z_{i j}^{1}=\frac{b_{i j}-\mu_{h}}{2} .
$$

The result can be deduced similarly for $Z_{i j}^{2}$. We can also show that $Z_{i j}^{2}=-Z_{i j}^{1}$, and satisfies

$$
b_{i j}\left(1-\epsilon D_{-}^{j} Z_{i j}^{2}\right)=\frac{b_{i j}+\mu_{h}}{2}, \quad-\epsilon a_{i j} D_{-}^{i} Z_{i j}^{2}=\frac{a_{i j}-\mu_{h}}{2} .
$$

This proves the lemma.

Remark. The explicit forms of $Z_{i j}^{1}$ and $Z_{i j}^{2}$ depend on $a_{\epsilon}(x-y)$ being a function of $x-y$. For the general angular dependences $a_{\epsilon}(\alpha x+\beta y)$, these forms would not be possible.

Lemma 3.2 Assume discrete function $\eta_{i j}$ defined as

$$
\eta_{i j}=\frac{h}{\epsilon}\left(\sum_{k=1}^{i} a_{k j}-i a_{h}\right)+\frac{h}{\epsilon}\left(j a_{h}-\sum_{k=1}^{j} b_{0 k}\right), \quad(i, j) \in \bar{\Omega}_{h} .
$$

Then, $\eta_{i j}$ is bounded, and has the following properties

$$
\epsilon D_{-}^{i} \eta_{i j}=a_{i j}-a_{h}, \quad \epsilon D_{-}^{j} \eta_{i j}=a_{h}-b_{i j}, \quad(i, j) \in \Omega_{h}
$$

Proof. Using the symmetric properties of the coefficients, we can establish the results similarly as in Lemma 3.1.

Lemma 3.3 Assume $U_{i j}$ satisfies

$$
\begin{aligned}
L_{\mu, h} U_{i j} & =\lambda_{\epsilon} \phi_{i j}^{h}, \quad(i, j) \in \Omega_{h}, \\
U_{i j} & =0, \quad(i, j) \in \partial \Omega_{h},
\end{aligned}
$$


where $\left(\lambda_{\epsilon}, \phi_{i j}^{h}\right)$ is a normalized eigenpair of $L_{\epsilon, h}$. Then, we have

$$
\|U\|_{1}^{2}=\sum_{i, j=0}^{N-1}\left[\left(D_{+}^{i} U_{i j} h\right)^{2}+\left(D_{+}^{j} U_{i j} h\right)^{2}\right]=O\left(\lambda_{\epsilon}\right) ;
$$

and

$$
\begin{aligned}
& \sum_{i, j=1}^{N-1}\left(\left(D_{+}^{i} D_{-}^{i} U_{i j}\right)^{2}+\left(D_{-}^{i} D_{-}^{j} U_{i j}\right)^{2}+\left(D_{+}^{i} D_{+}^{j} U_{i j}\right)^{2}+\left(D_{+}^{j} D_{-}^{j} U_{i j}\right)^{2}\right) h^{2} \\
& =O\left(\lambda_{\epsilon}^{2}\right) .
\end{aligned}
$$

Proof. First, we observe that

$$
\sum_{i, j=0}^{N-1}\left[\left(D_{+}^{i} \phi_{i j}^{h} h\right)^{2}+\left(D_{+}^{j} \phi_{i j}^{h} h\right)^{2}\right]=O\left(\lambda_{\epsilon}\right) .
$$

Applying $U_{i j}$ to the following equation and taking summation, we have

$$
\sum_{i, j=1}^{N-1} L_{\mu, h} U_{i j} U_{i j}=\sum_{i, j=1}^{N-1} L_{\epsilon, h} \phi_{i j} U_{i j} .
$$

(3.11) then follows. For (3.12), since $U_{i j}$ vanishes at the boundary, it satisfies

$$
\begin{aligned}
& \frac{1}{2} \sum_{i, j=1}^{N-1}\left[\left(D_{+}^{i} D_{-}^{i} U_{i j}\right)^{2}+\left(D_{+}^{j} D_{-}^{j} U_{i j}\right)^{2}\right] \geq \sum_{i, j=1}^{N-1}\left(D_{+}^{i} D_{-}^{i} U_{i j}\right)\left(D_{+}^{j} D_{-}^{j} U_{i j}\right) \\
= & \sum_{i, j=1}^{N}\left(D_{-}^{i} D_{-}^{j} U_{i j}\right)^{2}=\sum_{i, j=0}^{N-1}\left(D_{+}^{i} D_{+}^{j} U_{i j}\right)^{2} .
\end{aligned}
$$

Multiplying $D_{+}^{i} D_{-}^{i} U_{i j}$ to $(3.10)$, we get

$$
\begin{aligned}
& \left(D_{+}^{i} D_{-}^{i} U_{i j}\right)^{2}+2 \frac{a_{h}-\mu_{h}}{a_{h}+\mu_{h}}\left(D_{+}^{i} D_{+}^{j} U_{i j}\right)\left(D_{+}^{i} D_{-}^{i} U_{i j}\right)+\left(D_{+}^{i} D_{+}^{j} U_{i j}\right)^{2}+ \\
& \left(D_{+}^{i} D_{-}^{i} U_{i j}\right)^{2}+2 \frac{a_{h}-\mu_{h}}{a_{h}+\mu_{h}}\left(D_{-}^{i} D_{-}^{j} U_{i j}\right)\left(D_{+}^{i} D_{-}^{i} U_{i j}\right)+\left(D_{-}^{i} D_{-}^{j} U_{i j}\right)^{2}+ \\
& \left(\left(D_{+}^{j} D_{-}^{j} U_{i j}\right)\left(D_{+}^{i} D_{-}^{i} U_{i j}\right)-\left(D_{+}^{i} D_{+}^{j} U_{i j}\right)^{2}\right)+ \\
& \left(\left(D_{+}^{j} D_{-}^{j} U_{i j}\right)\left(D_{+}^{i} D_{-}^{i} U_{i j}\right)-\left(D_{-}^{i} D_{-}^{j} U_{i j}\right)^{2}\right) \\
= & \frac{2}{a_{h}+\mu_{h}} \lambda_{\epsilon} \phi_{i j}^{h} D_{+}^{i} D_{-}^{i} U_{i j}, \quad(i, j) \in \Omega_{h} .
\end{aligned}
$$


By the uniformly ellipticity property of the homogenized operator (3.4), we have

$$
\sum_{i, j=1}^{N-1}\left(\left(D_{+}^{i} D_{-}^{i} U_{i j}\right)^{2}+\left(D_{+}^{i} D_{+}^{j} U_{i j}\right)^{2}+\left(D_{-}^{i} D_{-}^{j} U_{i j}\right)^{2}\right) h^{2}=O\left(\lambda_{\epsilon}^{2}\right) .
$$

An similar argument gives

$$
\sum_{i, j=1}^{N-1}\left(\left(D_{+}^{j} D_{-}^{j} U_{i j}\right)^{2}+\left(D_{+}^{i} D_{+}^{j} U_{i j}\right)^{2}+\left(D_{-}^{i} D_{-}^{j} U_{i j}\right)^{2}\right) h^{2}=O\left(\lambda_{\epsilon}^{2}\right) .
$$

(3.13), (3.14) and (3.15) complete the proof of lemma.

Lemma 3.4 Assume that $U_{i j}$ defined in Lemma 3.3 satisfies the following boundary conditions,

$$
D_{+}^{i} U_{i j}+D_{-}^{i} U_{i j}=D_{+}^{j} U_{i j}+D_{-}^{j} U_{i j}=0, \quad(i, j) \in \partial \Omega_{h}
$$

Then,

$$
\begin{aligned}
\|U\|_{2}^{2} & =\sum_{i, j=0}^{N}\left(\left(D_{+}^{i} D_{-}^{i} U_{i j}\right)^{2}+\left(D_{+}^{j} D_{-}^{j} U_{i j}\right)^{2}\right) h^{2} \\
& +\sum_{i, j=0}^{N-1}\left(D_{+}^{i} D_{+}^{j} U_{i j} h\right)^{2}+\sum_{i, j=1}^{N}\left(D_{-}^{i} D_{-}^{j} U_{i j} h\right)^{2}=O\left(\lambda_{\epsilon}^{2} / h\right) .
\end{aligned}
$$

Proof. Since

$$
D_{-}^{i} U_{N j}=D_{-}^{i} U_{i j}+h \sum_{k=i}^{N-1} D_{+}^{i} D_{-}^{i} U_{k j}
$$

we have

$$
\left(D_{-}^{i} U_{N j}\right)^{2} \leq\left(D_{-}^{i} U_{i j}\right)^{2}+\sum_{i=1}^{N-1}\left(D_{+}^{i} D_{-}^{i} U_{i j}\right)^{2} h
$$

Then,

$$
\sum_{j=1}^{N}\left(D_{-}^{i} U_{N j}\right)^{2} h \leq \sum_{i, j=1}^{N}\left(D_{-}^{i} U_{i j} h\right)^{2}+\sum_{i, j=1}^{N}\left(D_{+}^{i} D_{-}^{i} U_{i j} h\right)^{2}=O\left(\lambda_{\epsilon}^{2}\right) .
$$

Combined (3.12) with the following relation,

$$
D_{+}^{i} D_{-}^{i} U_{N j}=-\frac{2}{h} D_{+}^{i} U_{N-1 j},
$$

the rest of the proof follows. 
Theorem 3.2 Assume $\phi_{i j}^{h}, U_{i j}$ are defined in Lemma 3.3 and Lemma 3.4. Then,

$$
\left\|\phi^{h}-U\right\|_{h}=O\left(\sqrt{\epsilon} \lambda_{\epsilon}\right)
$$

Proof. First, we introduce the following discrete functions

$$
\begin{aligned}
G_{i j} & =G_{i j}^{1}+G_{i j}^{2}, \\
G_{i j}^{1} & =\frac{1}{2} U_{i j}-\frac{\epsilon}{2}\left(Z_{i j}^{1} D_{+}^{i} U_{i j}+Z_{i j}^{2} D_{+}^{j} U_{i j}\right)-\frac{1}{2} \phi_{i j}^{h}, \\
G_{i j}^{2} & =\frac{1}{2} U_{i j}-\frac{\epsilon}{2}\left(Z_{i j}^{1} D_{-}^{i} U_{i j}+Z_{i j}^{2} D_{-}^{j} U_{i j}\right)-\frac{1}{2} \phi_{i j}^{h},
\end{aligned}
$$

for $(i, j) \in \bar{\Omega}_{h}$. By the assumption (3.16), $G_{i j}$ vanishes at boundary, i.e.,

$$
G_{i j}=0, \quad(i, j) \in \partial \Omega_{h} .
$$

For $G_{i j}^{1}$, we have

$$
\begin{aligned}
2 D_{-}^{i} G_{i j}^{1}= & D_{-}^{i} U_{i j}-\epsilon\left(D_{-}^{i}\left(Z_{i j}^{1} D_{+}^{i} U_{i j}\right)+D_{-}^{i}\left(Z_{i j}^{2} D_{+}^{j} U_{i j}\right)\right)-D_{-}^{i} \phi_{i j}^{h} \\
= & \left(D_{-}^{i} U_{i j}-\epsilon D_{-}^{i} Z_{i j}^{1} D_{+}^{i} U_{i-1 j}\right)-\epsilon Z_{i j}^{1} D_{-}^{i} D_{+}^{i} U_{i j} \\
& -\epsilon D_{-}^{i} Z_{i j}^{2} D_{+}^{j} U_{i j}-\epsilon Z_{i-1 j}^{2} D_{-}^{i} D_{+}^{j} U_{i j}-D_{-}^{i} \phi_{i j}^{h}, \\
2 D_{-}^{j} G_{i j}^{1}= & D_{-}^{j} U_{i j}-\epsilon\left(D_{-}^{j}\left(Z_{i j}^{1} D_{+}^{i} U_{i j}\right)+D_{-}^{j}\left(Z_{i j}^{2} D_{+}^{j} U_{i j}\right)\right)-D_{-}^{j} \phi_{i j}^{h} \\
= & \left(D_{-}^{j} U_{i j}-\epsilon D_{-}^{j} Z_{i j}^{2} D_{+}^{j} U_{i j-1}\right)-\epsilon Z_{i j}^{2} D_{-}^{j} D_{+}^{j} U_{i j} \\
& -\epsilon D_{-}^{j} Z_{i j}^{1} D_{+}^{i} U_{i j}-\epsilon Z_{i j-1}^{1} D_{-}^{j} D_{+}^{i} U_{i j}-D_{-}^{j} \phi_{i j}^{h} .
\end{aligned}
$$

Then,

$$
\begin{aligned}
2 L_{\epsilon, h} G_{i j}^{1}= & D_{+}^{i} \frac{a_{i j}+\mu_{h}}{2} D_{-}^{i} U_{i j}+D_{+}^{i} \frac{a_{i j}-\mu_{h}}{2} D_{+}^{j} U_{i j} \\
& -\epsilon D_{+}^{i}\left(a_{i j} Z_{i j}^{1} D_{+}^{i} D_{-}^{i} U_{i j}+a_{i j} Z_{i-1 j}^{2} D_{-}^{i} D_{+}^{j} U_{i j}\right) \\
& +D_{+}^{i} \frac{b_{i j}-\mu_{h}}{2} D_{+}^{j} U_{i j}+D_{+}^{j} \frac{b_{i j}+\mu_{h}}{2} D_{-}^{j} U_{i j} \\
& -\epsilon D_{+}^{j}\left(b_{i j} Z_{i j}^{2} D_{-}^{j} D_{+}^{j} U_{i j}+b_{i j} Z_{i j-1}^{1} D_{-}^{j} D_{+}^{i} U_{i j}\right) \\
& +L_{\epsilon} \phi_{i j}^{h} .
\end{aligned}
$$


For $G_{i j}^{2}$, we establish similarly

$$
\begin{aligned}
2 D_{+}^{i} G_{i j}^{2}= & D_{+}^{i} U_{i j}-\epsilon\left(D_{+}^{i}\left(Z_{i j}^{1} D_{-}^{i} U_{i j}\right)+D_{+}^{i}\left(Z_{i j}^{2} D_{-}^{j} U_{i j}\right)\right)-D_{+}^{i} \phi_{i j}^{h} \\
= & \left(D_{+}^{i} U_{i j}-\epsilon D_{+}^{i} Z_{i j}^{1} D_{-}^{i} U_{i+1 j}\right)-\epsilon Z_{i j}^{1} D_{-}^{i} D_{+}^{i} U_{i j} \\
& -\epsilon D_{+}^{i} Z_{i j}^{2} D_{-}^{j} U_{i j}-\epsilon Z_{i+1 j}^{2} D_{+}^{i} D_{-}^{j} U_{i j}-D_{+}^{i} \phi_{i j}^{h}, \\
2 D_{+}^{j} G_{i j}^{2}= & D_{+}^{j} U_{i j}-\epsilon\left(D_{+}^{j}\left(Z_{i j}^{1} D_{-}^{i} U_{i j}\right)+D_{+}^{j}\left(Z_{i j}^{2} D_{-}^{j} U_{i j}\right)\right)-D_{+}^{j} \phi_{i j}^{h} \\
= & \left(D_{+}^{j} U_{i j}-\epsilon D_{+}^{j} Z_{i j}^{2} D_{-}^{j} U_{i j+1}\right)-\epsilon Z_{i j}^{2} D_{+}^{j} D_{-}^{j} U_{i j} \\
& -\epsilon D_{+}^{j} Z_{i j}^{1} D_{-}^{i} U_{i j}-\epsilon Z_{i j+1}^{1} D_{+}^{j} D_{-}^{i} U_{i j}-D_{+}^{j} \phi_{i j}^{h} .
\end{aligned}
$$

Then,

$$
\begin{aligned}
2 L_{\epsilon, h} G_{i j}^{2}= & D_{-}^{i} \frac{a_{i+1 j}+\mu_{h}}{2} D_{+}^{i} U_{i j}+D_{-}^{i} \frac{a_{i+1 j}-\mu_{h}}{2} D_{+}^{j} U_{i j} \\
& +D_{-}^{j} \frac{b_{i j+1}-\mu_{h}}{2} D_{-}^{i} U_{i j}+D_{-}^{j} \frac{b_{i j+1}+\mu_{h}}{2} D_{+}^{j} U_{i j} \\
& -\epsilon D_{-}^{i}\left(a_{i+1 j} Z_{i j}^{1} D_{-}^{i} D_{+}^{i} U_{i j}+a_{i+1 j} Z_{i+1 j}^{2} D_{+}^{i} D_{-}^{j} U_{i j}\right) \\
& -\epsilon D_{-}^{j}\left(b_{i j+1} Z_{i j}^{2} D_{-}^{j} D_{+}^{j} U_{i j}+b_{i j+1} Z_{i j+1}^{1} D_{+}^{j} D_{-}^{i} U_{i j}\right) \\
& +L_{\epsilon} \phi_{i j}^{h} .
\end{aligned}
$$

For simplicity, we introduce another two operators, $L_{1}$ and $L$ by

$$
L_{1}=\frac{a_{i j}+b_{i j}-2 a_{h}}{2}\left(D_{+}^{i} D_{-}^{i}+D_{+}^{i} D_{+}^{j}+D_{-}^{i} D_{-}^{j}+D_{+}^{j} D_{-}^{j}\right),
$$

and

$$
\begin{aligned}
& L=\frac{1}{2}( \\
& D_{+}^{i} \frac{a_{i j}+\mu_{h}}{2} D_{-}^{i}+D_{-}^{i} \frac{a_{i+1 j}-\mu_{h}}{2} D_{-}^{j}+D_{-}^{j} \frac{b_{i j+1}-\mu_{h}}{2} D_{-}^{i}+D_{+}^{j} \frac{b_{i j}+\mu_{h}}{2} D_{-}^{j} \\
& \left.+\quad D_{-}^{i} \frac{a_{i+1 j}+\mu_{h}}{2} D_{+}^{i}+D_{+}^{i} \frac{a_{i j}-\mu_{h}}{2} D_{+}^{j}+D_{+}^{j} \frac{b_{i j}-\mu_{h}}{2} D_{+}^{i}+D_{-}^{j} \frac{b_{i j+1}+\mu_{h}}{2} D_{+}^{j}\right) .
\end{aligned}
$$

Observe that

$$
L_{\epsilon, h} \phi_{i j}^{h}=L_{\mu, h} U_{i j}=L U_{i j}-L_{1} U_{i j}, \quad(i, j) \in \Omega_{h},
$$


from (3.19), (3.20) and (3.21), we get

$$
\begin{gathered}
2 L_{\epsilon, h} G_{i j}=2 L_{\epsilon, h} G_{i j}^{1}+2 L_{\epsilon, h} G_{i j}^{2}=-2 L_{1} U_{i j} \\
-\quad \epsilon D_{+}^{i}\left(a_{i j} Z_{i j}^{1} D_{+}^{i} D_{-}^{i} U_{i j}+a_{i j} Z_{i-1 j}^{2} D_{-}^{i} D_{+}^{j} U_{i j}\right) \\
-\quad \epsilon D_{+}^{j}\left(b_{i j} Z_{i j}^{2} D_{-}^{j} D_{+}^{j} U_{i j}+b_{i j} Z_{i j-1}^{1} D_{-}^{j} D_{+}^{i} U_{i j}\right) \\
-\quad \epsilon D_{-}^{i}\left(a_{i+1 j} Z_{i j}^{1} D_{-}^{i} D_{+}^{i} U_{i j}+a_{i+1 j} Z_{i+1 j}^{2} D_{+}^{i} D_{-}^{j} U_{i j}\right) \\
-\epsilon D_{-}^{j}\left(b_{i j+1} Z_{i j}^{2} D_{-}^{j} D_{+}^{j} U_{i j}+b_{i j+1} Z_{i j+1}^{1} D_{+}^{j} D_{-}^{i} U_{i j}\right) .
\end{gathered}
$$

Meanwhile, by Lemma 3.2, taking summations by parts, we have

$$
\begin{aligned}
& \sum_{i, j=1}^{N-1}\left(a_{i j}-a_{h}\right)\left(D_{+}^{j} D_{-}^{j} U_{i j}\right) G_{i j}=\epsilon \sum_{i, j=1}^{N-1} D_{-}^{i} \eta_{i j} D_{+}^{j} D_{-}^{j} U_{i j} G_{i j} \\
= & -\epsilon \sum_{i, j=1}^{N} \eta_{i-1 j} D_{-}^{i}\left(D_{+}^{j} D_{-}^{j} U_{i j} G_{i j}\right) \\
= & -\epsilon \sum_{i, j=1}^{N} \eta_{i-1 j} D_{-}^{i} D_{+}^{j} D_{-}^{j} U_{i j} G_{i-1 j}-\epsilon \sum_{i, j=1}^{N} \eta_{i-1 j} D_{+}^{j} D_{-}^{j} U_{i j} D_{-}^{i} G_{i j} \\
= & -\epsilon \sum_{i, j=1}^{N} \eta_{i j} D_{+}^{i} D_{-}^{j} D_{+}^{j} U_{i j} G_{i j}-\epsilon \sum_{i, j=1}^{N} \eta_{i-1 j} D_{+}^{j} D_{-}^{j} U_{i j} D_{-}^{i} G_{i j} \\
= & \epsilon \sum_{i, j=1}^{N} D_{-}^{j}\left(\eta_{i j} G_{i j}\right) D_{+}^{i} D_{+}^{j} U_{i j-1}-\epsilon \sum_{i, j=1}^{N} \eta_{i-1 j} D_{+}^{j} D_{-}^{j} U_{i j} D_{-}^{i} G_{i j} \\
= & \epsilon \sum_{i, j=0}^{N-1} D_{+}^{j}\left(\eta_{i j} G_{i j}\right) D_{+}^{i} D_{+}^{j} U_{i j}-\epsilon \sum_{i, j=1}^{N} \eta_{i-1 j} D_{+}^{j} D_{-}^{j} U_{i j} D_{-}^{i} G_{i j} \\
= & \epsilon \sum_{i, j=0}^{N-1} D_{+}^{j} \eta_{i j} D_{+}^{i} D_{+}^{j} U_{i j} G_{i j}+\epsilon \sum_{i, j=0}^{N-1} \eta_{i j+1} D_{+}^{j} G_{i j} D_{+}^{i} D_{+}^{j} U_{i j} \\
& -\epsilon \sum_{i, j=1}^{N} \eta_{i-1 j} D_{+}^{j} D_{-}^{j} U_{i j} D_{-}^{i} G_{i j} \\
& \quad \sum_{i, j=0}^{N-1}\left(a_{h}-b_{i j+1}\right) D_{+}^{i} D_{+}^{j} U_{i j} G_{i j}+\epsilon \sum_{i, j=0}^{N-1} \eta_{i j+1} D_{+}^{j} G_{i j} D_{+}^{i} D_{+}^{j} U_{i j} \\
& -\epsilon \sum_{i, j=1}^{N} \eta_{i-1 j} D_{+}^{j} D_{-}^{j} U_{i j} D_{-}^{i} G_{i j} . \\
= &
\end{aligned}
$$


By the symmetric property of the coefficients, $a_{i j}=b_{i j+1}$, we get

$$
\begin{aligned}
& \sum_{i, j=1}^{N-1}\left(a_{i j}-a_{h}\right)\left(D_{+}^{j} D_{-}^{j}+D_{+}^{i} D_{+}^{j}\right) U_{i j} G_{i j} \\
= & \epsilon \sum_{i, j=0}^{N-1} \eta_{i j+1} D_{+}^{j} G_{i j} D_{+}^{i} D_{+}^{j} U_{i j}-\epsilon \sum_{i, j=1}^{N} \eta_{i-1 j} D_{+}^{j} D_{-}^{j} U_{i j} D_{-}^{i} G_{i j} .
\end{aligned}
$$

Proceeding in the same way as before, we obtain

$$
\begin{aligned}
& \sum_{i, j=1}^{N-1}\left(b_{i j}-a_{h}\right)\left(D_{+}^{j} D_{-}^{j}+D_{-}^{i} D_{-}^{j}\right) U_{i j} G_{i j} \\
= & -\epsilon \sum_{i, j=1}^{N} \eta_{i j} D_{-}^{i} G_{i j} D_{+}^{j} D_{-}^{j} U_{i-1 j}+\epsilon \sum_{i, j=0}^{N-1} \eta_{i j} D_{-}^{i} D_{-}^{j} U_{i j+1} D_{+}^{j} G_{i j} \\
& \sum_{i, j=1}^{N-1}\left(a_{i j}-a_{h}\right)\left(D_{+}^{i} D_{-}^{i}+D_{-}^{i} D_{-}^{j}\right) U_{i j} G_{i j} \\
= & \epsilon \sum_{i, j=0}^{N-1} \eta_{i j+1} D_{+}^{j} G_{i j} D_{+}^{i} D_{-}^{i} U_{i j}-\epsilon \sum_{i, j=0}^{N-1} \eta_{i j} D_{-}^{i} D_{-}^{j} U_{i+1 j} D_{+}^{i} G_{i j} . \\
& \sum_{i, j=1}^{N-1}\left(b_{i j}-a_{h}\right)\left(D_{+}^{i} D_{-}^{i}+D_{+}^{i} D_{+}^{j}\right) U_{i j} G_{i j} \\
= & \epsilon \sum_{i, j=1}^{N} \eta_{i j-1} D_{-}^{j} G_{i j} D_{+}^{i} D_{-}^{i} U_{i j}-\epsilon \sum_{i, j=1}^{N} \eta_{i j} D_{+}^{i} D_{+}^{j} U_{i-1 j} D_{-}^{i} G_{i j} .
\end{aligned}
$$

Combining (3.23), (3.24), (3.25) and (3.26), we get

$$
\begin{aligned}
& \sum_{i, j=1}^{N} \frac{a_{i j}+b_{i j}-2 a_{h}}{2}\left(D_{+}^{j} D_{-}^{j}+D_{-}^{i} D_{-}^{j}+D_{+}^{i} D_{-}^{i}+D_{+}^{i} D_{+}^{j}\right) U_{i j} G_{i j} h^{2} \\
\leq & C \epsilon\|U\|_{2} \sqrt{\sum_{i, j=0}^{N-1}\left[\left(D_{+}^{i} G_{i j} h\right)^{2}+\left(D_{+}^{j} G_{i j} h\right)^{2}\right]} \\
\leq & C \sqrt{\epsilon} \lambda_{\epsilon} \sqrt{\sum_{i, j=0}^{N-1}\left[\left(D_{+}^{i} G_{i j} h\right)^{2}+\left(D_{+}^{j} G_{i j} h\right)^{2}\right]}
\end{aligned}
$$


Further,

$$
\begin{aligned}
& \epsilon \sum_{i, j=0}^{N-1}\left[D_{+}^{i}\left(a_{i j} Z_{i j}^{1} D_{+}^{i} D_{-}^{i} U_{i j}+a_{i j} Z_{i-1 j}^{2} D_{-}^{i} D_{+}^{j} U_{i j}\right)\right] G_{i j} h^{2} \\
& =\epsilon \sum_{i, j=0}^{N-1}\left[a_{i+1 j} Z_{i+1 j}^{1} D_{+}^{i} D_{-}^{i} U_{i+1 j}+a_{i+1 j} Z_{i j}^{2} D_{-}^{i} D_{+}^{j} U_{i+1 j}\right] D_{+}^{i} G_{i j} h^{2} \\
& \leq C \epsilon \sqrt{\sum_{i, j=0}^{N-1}\left(D_{+}^{i} D_{-}^{i} U_{i+1 j}\right)^{2} h^{2}}+\sqrt{\left.\sum_{i, j=0}^{N-1}\left(D_{+}^{i} D_{+}^{j} U_{i j}\right)^{2} h^{2}\right)} \sqrt{\sum_{i, j=0}^{N-1}\left(D_{+}^{i} G_{i j}\right)^{2} h^{2}} \\
& =C \sqrt{\epsilon} \lambda_{\epsilon} \sqrt{\sum_{i, j=0}^{N-1}\left(D_{+}^{i} G_{i j}\right)^{2} h^{2} .}
\end{aligned}
$$

The exact same order for the last three terms in (3.22) can be established similarly. Consequently, from (3.27) and (3.28),

$$
\sqrt{\sum_{i, j=1}^{N}\left(D_{-}^{i} G_{i j} h\right)^{2}+\left(D_{-}^{j} G_{i j} h\right)^{2}}=O\left(\sqrt{\epsilon} \lambda_{\epsilon}\right) .
$$

By Poincare inequality,

$$
\|G\|_{h}=O\left(\sqrt{\epsilon} \lambda_{\epsilon}\right)
$$

By Lemma 3.1 and Lemma 3.3,

$$
\left\|\epsilon Z^{1}\left(D_{0}^{i} U-D_{0}^{j} U\right)\right\|_{h} \leq \epsilon \max _{i, j \in \bar{\Omega}_{h}}\left|Z_{i j}^{1}\right|\|U\|_{1}=O\left(\epsilon \lambda_{\epsilon}\right)
$$

which implies,

$$
\left\|\left(I-L_{\mu, h}^{-1} L_{\epsilon, h}\right) \phi^{h}\right\|_{h} \leq C \sqrt{\epsilon} \lambda_{\epsilon} .
$$

We hence complete the proof.

Remark. The result in (3.18) here is consistent with the result established in [8] for the continuous case.

Proof of Theorem 3.1. The procedure is exactly the same as that in Theorem 2.1, except different inequalities estimated. By Theorem 3.2, we have

$$
I_{1} \leq C \sqrt{h} k_{0}^{3},
$$


instead of $(2.20)$. Therefore, in order to make $I_{1}<\frac{1}{2}$, we set

$$
k_{0} \leq C h^{-1 / 6}
$$

instead of (2.21). For $I_{2} \leq \frac{1}{2}$, it is sufficient to have

$$
\gamma \geq C \frac{h^{-2}}{k_{0}^{2}} \ln k_{0}\left(\geq \frac{1}{\alpha k_{0}^{2}}\right)
$$

Combining (3.31) and (3.32), we have

$$
\gamma \geq C h^{-1-\frac{2}{3}} \ln h
$$

As what we have done in previous section, we consequently establish the following main Theorem.

Theorem 3.3 There exists constant $C$, the operator $M$ defined by (2.5) satisfies

$$
\rho(M) \leq \rho_{0}<1,
$$

whenever the step size $h$ belongs to the set $S\left(\epsilon, h_{0}\right)$ of Diophantine numbers, and

$$
\gamma \geq C h^{-1-2 / 3} \ln h
$$

\section{Conclusion}

The analysis of the proof strongly indicates us the role of homogenization, which plays in the convergence process. If, for example, the coarse grid operator is replaced by its averaged operator in one dimensional problem [5], the direct estimate for multigrid convergence rate is not asymptotically better than just using the damped Jacobi smoothing operator. This follows from the effect of the oscillations on the low eigenmodes. The homogenized coarse grid operator reduces the number of smoothing operation from $O\left(h^{-2}\right)$ to $O\left(h^{-6 / 5} \ln h\right)$, when the step size $h$ belongs to the set $S\left(\epsilon, h_{0}\right)$ of Diophantine numbers. In [9], it has also been shown that the number of smoothing iteration needed for the convergence of the multigird method with the average coarse grid operator guarantees the one with the homogenized coarse grid operator.

The theoretical results established in this paper seem a little bit disappointing. From a number of numerical experiments $[5,6]$, we can get much faster convergence rate in practice 
than that required in the theoretical results. However, numerical results do indicate that the convergent rate depends on the grid size $h$ for these types of equations with oscillatory coefficients $[5,6]$.

There are some inequalities in the implementation of the proof, which are potential to be improved so that a sharper convergent rate is possible. One of them is to enlarge the space of low eigenmodes, which can be approximated by the corresponding homogenized eigenmodes. Such as to improve (3.18) to (2.14), which we think is the sharpest inequality one can establish. We established the same inequality for the one dimensional case as (2.14) in the two dimensional case oscillatory along a coordinate direction [9]. However, the portion of the eigenmodes that can be approximated by the homogenized ones in later case is relatively much smaller than the previous one. That's why we obtain $O\left(h^{-4 / 3} \ln h\right)$ for the number of smoothing iterations instead of $O\left(h^{-6 / 5} \ln h\right)$ for the later case, although there have the same inequality (2.14) for the space of low eigenmodes.

Nevertheless, from the analysis of homogenization, we understand that there always exists a boundary layer $[2,10]$, which makes it hard to get the first lower order correction of the eigenfunctions. The case we discussed in chapter 2, which is equivalently to one dimensional problem, doesn't have such a boundary layer. We hence get an estimate as in (2.14). For the case in chapter 3, all we can establish is (3.18), which consists of the result established in [8] for the continuous case. And, it also defines us a smaller low eigenspace. However, numerical examples tells us that there are also some difference between these two cases. That a complete understanding of the first lower order correction for the eigenfunctions is required to further improve the estimates.

\section{References}

[1] R.E. Alcouffe, A. Brandt, J.E. Dendy, AND J.W. Painter, The Multi-Grid Method for the Diffusion Equation with Strongly Discontinuous Coefficients, SIAM J. Sci. Stat. Comput., Vol. 2, No. 4 (1981), pp. 430-454.

[2] A. Bensoussan, J.L. Lions, AND G. Papanicolaou, Asymptotic Analysis for Periodic Structure, Studies in Mathematics and Its Applications, Vol. 5, North-Holland Publ., 1978 .

[3] A. Brandt, Multi-level Adaptive Solutions to Boundary-Value Problems, Mathematics of Computation, Vol. 31, No. 136 (1977), pp.333-390. 
[4] B. Engquist, Computation of Oscillatory Solutions for Partial Differential Equations, Lecture Notes in Mathematics 1270 (1989), pp. 10-22.

[5] B. Engquist AND E. Luo: Multigrid Methods For Differential Equations With Highly Oscillatory Coefficients. Proceedings of the Sixth Copper Mountain Conference on Multigrid Methods, 1993, pp. 175-190.

[6] B. Engquist AND E. Luo: New Coarse Grid Operators of Multigrid Methods for Highly Oscillatory Coefficient Elliptic Problems. Preprint.

[7] L. Giraud AND R.S. Tuminaro: Grid Transfer Operators for Highly Variable Coefficient Problems. Preprint.

[8] S. Kesavan, Homogenization of Elliptic Eigenvalue Problems: Part 1, Applied Mathematics and Optimization, Vol. 5, (1979), pp.153-167.

[9] E. Luo: Multigrid Method for Elliptic Equation with Oscillatory Coefficients. Ph.D. Thesis, UCLA, 1993.

[10] F. Santosa AND M. Vogelius: First Order Corrections to the Homogenized Eigenvalues of a Periodic Composite Medium. SIAM J. Appl. Math., vol. 53, no. 6, 1993, pp. 16361668.

[11] W. Hackbusch, Multi-Grid Methods and Applications, Berlin; New York: springerVerlag, 1985.

[12] W. Hackbusch AND U. Trottenburg (eds), Multigrid Methods, Proceedings, KolnPolrz, Lecture Notes in Mathematics 960, Berlin; New York: springer-Verlag. 